\title{
A POLIGERAÇÃO NA PRODUÇÃO DE BIOCOMBUSTÍVEIS: ETANOL E BIOMETANOL
}

\author{
Maria Luiza Grillo Renó ${ }^{1}$ \\ José Carlos Escobar Palacio ${ }^{2}$ \\ Electo Eduardo Silva Lora ${ }^{3}$
}

\begin{abstract}
Resumo
Neste trabalho são apresentadas duas rotas tecnológicas de produção de biocombustível provenientes da cana-de-açúcar no contexto da poligeração. O primeiro caso consiste numa destilaria autônoma de produção de etanol com uma capacidade de moagem de 380 toneladas de cana por hora, gerando excedentes elétricos para comercialização na rede a partir de seu sistema de cogeração baseado em turbinas de extração/condensação que opera com parâmetros de vapor de $6.5 \mathrm{MPa}$ e $490{ }^{\circ} \mathrm{C}$. No segundo caso será considerada uma planta de produção de biometanol a partir da gaseificação do bagaço de cana-de-açúcar acoplada a uma destilaria de etanol, com uma capacidade de produção 50.000 toneladas de biometanol por ano. As duas alternativas tecnológicas de produção foram analisadas a partir de indicadores baseados na primeira e segunda lei da termodinâmica, assim como alguns indicadores econômicos. Os resultados mostraram que a implementação dos sistemas de poligeração na produção de biocombustíveis é benéfico a sustentabilidade pois aumenta o uso eficiente dos recursos demandados pelos processos da planta.
\end{abstract}

Palavras Chaves:Biocombustíveis, Biometanol, Cogeração, Etanol.

\begin{abstract}
This work presents two technological routes of biofuel production from sugarcane in the context of polygeneration. The first case is an autonomous distillery to produce ethanol with a crushing capacity of 380 tonnes of sugarcane per hour, generating surplus electricity for marketing in the electrical network by its cogeneration system based on turbine extraction / condensation that operates with parameters steam of $6.5 \mathrm{MPa}$ and $490{ }^{\circ} \mathrm{C}$. In the second case will be considered a plant production of biomethanol by gasification of sugarcane bagasse that is coupled to an ethanol distillery, with a production capacity of 50,000 tons per year of biomethanol. The two technological alternatives were analyzed through indicators based on the first and second law of thermodynamics, as well as some economic indicators. The results showed that the implementation of polygeneration systems in biofuels production is benefict to the sustainability because increase the efficient use of resources demanded by the processes of the plant.
\end{abstract}

Keywords:Biofuels, Biomethanol, Cogeneration, Ethanol.

\footnotetext{
1 malureno@yahoo.com.br, Universidade Federal de Itajubá, Av. BPS 1303, CP 50, Itajubá-MG, Brasil

2jocescobar@gmail.com, Universidade Federal de Itajubá, Av. BPS 1303, CP 50, Itajubá-MG, Brasil

3electo@unifei.edu.br, Universidade Federal de Itajubá, Av. BPS 1303, CP 50, Itajubá-MG, Brasil
} 
Nomenclatura

\begin{tabular}{|c|c|}
\hline $\mathrm{B}$ & Bifurcação \\
\hline $\mathrm{B}_{\mathrm{f}}$ & Exergia do combustível \\
\hline $\mathrm{B}_{\mathrm{p}}$ & $\begin{array}{l}\text { Exergia consumida pelo } \\
\text { processo }\end{array}$ \\
\hline $\mathrm{c}_{\text {biocombustiveis }}$ & $\begin{array}{l}\text { Custo unitário dos } \\
\text { Biocombustíveis }\end{array}$ \\
\hline $\mathrm{c}_{\text {eletricidade }}$ & Custo unitário da eletricidade \\
\hline $\mathrm{C}_{\text {biocombustível }}$ & Custo do biocombustível \\
\hline $\mathrm{C}_{\mathrm{c}}$ & Custo da cana-de-açúcar \\
\hline $\mathrm{C}_{\text {eletricidade }}$ & Custo da eletricidade \\
\hline $\mathrm{E}_{\mathrm{b}}$ & Exergia do bagaço \\
\hline $\mathrm{E}_{\mathrm{bcog}}$ & $\begin{array}{l}\text { Exergia do bagaço destinado a } \\
\text { cogeração }\end{array}$ \\
\hline $\mathrm{E}_{\mathrm{bBM}}$ & $\begin{array}{l}\text { Exergia do bagaço destinado a } \\
\text { produção de biometanol }\end{array}$ \\
\hline $\mathrm{E}_{\text {caldo }}$ & $\begin{array}{l}\text { Exergia do caldo de } \\
\text { cana-de-açúcar }\end{array}$ \\
\hline $\mathrm{E}_{\text {cana }}$ & Exergia da cana-de-açúcar \\
\hline$E_{\operatorname{cog}}$ & Exergia para geração \\
\hline $\mathrm{E}_{\text {etanol }}$ & Exergia do etanol produzido \\
\hline $\mathrm{E}_{\text {metanol }}$ & Exergia do metanol \\
\hline$E+E$ & $\begin{array}{l}\text { Sistema de produção de } \\
\text { eletricidade + etanol }\end{array}$ \\
\hline $\mathrm{E}+\mathrm{E}+\mathrm{BM}$ & $\begin{array}{l}\text { Sistema de produção de } \\
\text { eletricidade }+ \text { etanol }+ \\
\text { biometanol }\end{array}$ \\
\hline FUE & Fator de Utilização de Energia \\
\hline $\mathrm{I}_{\text {exced }}$ & $\begin{array}{l}\text { Índice de eletricidade } \\
\text { excedente }\end{array}$ \\
\hline $\mathrm{J}$ & Junção \\
\hline $\mathrm{m}_{\mathrm{comb}}$ & $\begin{array}{l}\text { Fluxo total de bagaço } \\
\text { consumido pelas caldeiras }\end{array}$ \\
\hline PCI & Poder Calorífico Inferior \\
\hline $\mathrm{P}_{\mathrm{e}}$ & Potência elétrica líquida \\
\hline $\mathrm{P}_{\mathrm{ec}}$ & Potência elétrica consumida \\
\hline $\mathrm{Qu}_{\mathrm{u}}$ & $\begin{array}{l}\text { Fluxo de calor útil para } \\
\text { processo }\end{array}$ \\
\hline $\mathrm{R}$ & Fator de escala \\
\hline Sub & Subsistema \\
\hline $\mathrm{Tc}$ & Toneladas de cana moída \\
\hline$\dot{\mathrm{V}}_{\text {biocombustível }}$ & $\begin{array}{l}\text { Produção horária de } \\
\text { biocombustível }\end{array}$ \\
\hline $\mathrm{W}$ & $\begin{array}{l}\text { Potência produzida pelo } \\
\text { sistema }\end{array}$ \\
\hline $\mathrm{W}_{\text {exced }}$ & Excedente de energia elétrica \\
\hline $\mathrm{Z}_{\mathrm{PP}}$ & $\begin{array}{l}\text { Custo de amortização, operação } \\
\text { e manutenção dos equipamentos } \\
\text { daplanta }\end{array}$ \\
\hline$\eta_{\mathrm{e}}$ & Eficiência elétrica \\
\hline$\eta_{\text {exerg }}$ & Eficiência exergética \\
\hline$\eta_{\text {global }}$ & Eficiência global da planta \\
\hline
\end{tabular}

\section{INTRODUÇÃO}

Embora tenha grandes quantidades de petróleo ainda para serem exploradas, o Brasil precisa continuar estimulando a investigação de novas fontes e de novos processos de produção de combustíveis renováveis como forma de promover o desenvolvimento sustentável, criar empregos no campo e diversificar ainda mais a sua matriz energética.

OPró-Álcool foi um programa de sucesso e lançou as bases para que o país tenha atualmente um setor de biocombustíveis importante economicamente e reconhecido no mundo inteiro.Neste trabalho serão tratados dois biocombustíveis, o metanol e o etanol. O primeiro é produzido pela rota da gaseificação da biomassa, e o segundo pelo processo de fermentação alcoólica.

As principais características do processo produtivo destes dois biocombustíveis serão analisadas através de dois estudos de caso. $\mathrm{O}$ primeiro consiste numa destilaria autônoma convencional de produção de etanol, que gera excedentes elétricos pelo processo de cogeração com bagaço de cana-deaçúcar.

O segundo é considerado uma planta de produção de metanol, a partir da gaseificação do bagaço, acoplada a uma destilaria de etanol. Neste sistema a demanda térmica também será suprida pelo processo de cogeração com bagaço.

\section{REVISÃo BIBLIOGRÁFICA}

As principais características das rotas tecnológicas de produção dos biocombustíveis etanol e metanol, bem como da tecnologia de poligeração, estão apresentadas nos itens a seguir:

\subsection{Poligeração}

A poligeração é um conceito que se aplicado é muito vantajosodo ponto de vista energético, como também uma das soluções viáveis para as emissões excessivas dos gases de efeito estufa e o consumo elevado de combustíveis fósseis (Yamashita e Barreto, 2005).

A poligeração pode ser definida como a produção combinada de dois ou mais serviços energéticos ou produtos manufaturados, procurando atingir o 
máximo aproveitamento termodinâmico das fontes naturais utilizadas. Suas principais vantagens concentram-se em três aspectos:

- Eficiência energética: devido a forte integração entre geração de potência e síntese química, o aproveitamento energético de um sistema de poligeração é maior em relação a um sistema autônomo que produz os mesmos produtos.

- Combustíveis alternativos: produtos químicos produzidos por uma planta de poligeração podem ser usados para substituir combustíveis líquidos tradicionais, por exemplo, o metanol substituindo a gasolina, ou dimetil éter sendo usado no lugar de óleo diesel.

- Redução de emissões: um sistema energético poligeração em grande escala, normalmente apresenta tecnologias de captura do gás $\mathrm{CO}_{2}$.

- Redução dos custos: um sistema de poligeração tem como benefício a redução dos custos dos produtos finais.

Atualmente existem várias configurações propostas aos sistemas de poligeração, muitos deles focando análises econômica, energética e exergética destes sistemas.

No trabalho de Ma et al. (2004) foi proposto um sistema energético de poligeração que produz eletricidade e metanol, utilizando como insumo energético o carvão mineral. Através de uma análise econômica e da eficiência energética obtida pelo sistema, eles chegaram à conclusão da viabilidade do sistema.

Já em Chen et al. (2006) foi feita uma análise energética e de eficiência energética entre plantas de poligeração produzindo eletricidade e dimetil éter e plantas autônomas produzindo somente dimetil éter. Uma das conclusões obtidas foi uma economia energética de aproximadamente $17 \%$ para as plantas de poligeração.

\subsection{Biometanol}

Atualmente existem várias rotas comerciais para a produção de metanol, bem como estão em desenvolvimento tecnologias inovadoraspara a sua produção. De acordo com Cifre e Badr (2007) o processo de produção do metanol a partir da biomassa segue os seguintes passos:

\section{I - Pré-tratamento da matéria-} prima:

As fontes de biomassa mais apropriadas para esta aplicação são a madeira e os resíduos de madeira. A preparação da matéria-prima depende das características da biomassa e do gaseificador, e normalmente inclui a redução de tamanho das partículasda biomassa e a sua secagem.

\section{II - Gaseificação da biomassa:}

A gaseificação de biomassa consiste na sua transformação em gás de síntese, que é a matéria-prima da síntese catalítica de combustíveis e outros produtos. A seleção e projeto de umsistema de gaseificação de biomassa depende das características da matéria-prima, custos e desempenho do equipamento avaliado, como também da capacidade.

\section{III -Limpeza do gás de síntese:}

O gás produzido na gaseificação, também conhecido como gás de síntese ou syngas,é uma mistura de $\mathrm{CO}$ e $\mathrm{H}_{2}$, mas pode conter contaminante e por isso deve ser puficado antes de ser usado nos reatores de síntese catalítica. Ele pode conter alcatrão, partículas sólidas, álcalis, compostos de enxofre (geralmente a biomassa não tem enxofre) e cloro, os quais podem corroer o equipamento e também bloquear ou corromper a ação dos catalisadores.

O gás pode ser limpo usando tecnologias convencionais, como resfriamento, filtração em baixa temperatura, e lavadores utilizando água. Outra possibilidade é a limpeza do gás a quente, usando filtros de cerâmicas e diversos reagentes operando a 350 $800^{\circ} \mathrm{C}$.

\section{IV -Reforma dos Hidrocarbonetos:}

$\mathrm{Na}$ presença de catalisadores, metano, alcatrão e outros hidrocarbonetos são reformados até $\mathrm{CO}$ e $\mathrm{H}_{2}$ em altas temperaturas $\left(830-1000^{\circ} \mathrm{C}\right)$.

As reações apresentadas na Equação 
(1) representam o processo de reforma dos hidrocarbonetos, as reações são consideradas altamente endotérmicas e catalíticas (catalisador de níquel).

$$
\begin{aligned}
& \mathrm{CH}_{4}+\mathrm{H}_{2} \mathrm{O} \rightarrow \mathrm{CO}+3 \mathrm{H}_{2} \\
& \mathrm{C}_{2} \mathrm{H}_{4}+2 \mathrm{H}_{2} \mathrm{O} \rightarrow 2 \mathrm{CO}+4 \mathrm{H}_{2} \\
& \mathrm{C}_{2} \mathrm{H}_{6}+2 \mathrm{H}_{2} \mathrm{O} \rightarrow 2 \mathrm{CO}+5 \mathrm{H}_{2}
\end{aligned}
$$

A reforma é favorecida em baixas pressões, mas elevadas pressões beneficiam economicamente (equipamentos menores). Reformadores típicos operam entre 1 e $3.5 \mathrm{MPa}$, com uma temperatura entre $830^{\circ} \mathrm{C}$ e $1000^{\circ} \mathrm{C}$ (Maiyaet al., 2000).

\section{$\mathrm{V}$-Ajuste $\mathrm{H}_{2}$ /CO:}

$\mathrm{O}$ ajuste da relação $\mathrm{H}_{2} / \mathrm{CO}$ é realizado através da reação química do $\mathrm{CO}$ com $\mathrm{H}_{2} \mathrm{O}$, conforme mostrado na Equação (2).

Em experimentos laboratoriais tem-se alcançado bons resultados de síntese de metanol para uma relação $\mathrm{H}_{2} / \mathrm{CO}$ pouco maior que 2 (Maiyaet al., 2000).

$$
\mathrm{CO}+\mathrm{H}_{2} \mathrm{O} \leftrightarrow \mathrm{CO}_{2}+\mathrm{H}_{2}
$$

\section{VI - Síntese do Metanol:}

O metanol é produzido pela hidrogenação dos óxidos de carbono (Equação(3)) na presença de catalisadores que são baseados em óxido de cobre, óxido de zinco ou óxido de cromo (Ouelletteet al., 1995):

$$
\begin{aligned}
& \mathrm{CO}+2 \mathrm{H}_{2} \leftrightarrow \mathrm{CH}_{3} \mathrm{OH} \\
& \mathrm{CO}_{2}+3 \mathrm{H}_{2} \leftrightarrow \mathrm{CH}_{3} \mathrm{OH}+\mathrm{H}_{2} \mathrm{O}
\end{aligned}
$$

A primeira reação é a síntese do metanol primário. Uma pequena quantidade de $\mathrm{CO}_{2}$ na alimentação (2 $10 \%$ ) age como um promotor da segunda reação e ajuda a manter a atividade catalítica. A estequiometria de ambas as reações é satisfeita quando o coeficiente $\mathrm{R}$ (Equação (4)) apresenta um mínimo de 2.03.

$$
R=\frac{\mathrm{H}_{2}-\mathrm{CO}_{2}}{\mathrm{CO}+\mathrm{CO}_{2}}
$$

As reações de síntese do metanol são exotérmicas e levam a uma diminuição líquida no volume molar. Portanto o equilíbrio é favorecido pela alta pressão e baixa temperatura. $\mathrm{O}$ calor liberado deve ser removido para manter a catálise e taxa de reação.

\section{VII - Destilação:}

Depois do resfriamento e separação das impurezas do gás, o metanol cru é processado numa unidade de destilação para alcançar a qualidade requerida.

\subsection{Etanol de cana-de-açúcar}

O processo de produção de etanol a partir da cana-de-açúcar começa com o transporte da cana-de-açúcar da área de cultivo para a usina.

$\mathrm{Na}$ usina ocorre a lavagem da cana com água, sendo posteriormente transportada a um conjunto de facas rotativas e desfibradores, que preparam os caules para a moagem. Assim, a matéria-prima picada e já desfibrada é destinada às moendas para extração do caldo.

As próximas etapas do processo produtivo estão descritas a seguir (Santos, 2004):

Tratamento do caldo: $O$ caldo passa por um tratamento primário de peneiramento, depois é submetido a um tratamento mais completo que implica na adição de cal, aquecimento e posterior decantação.

Já com o caldo livre de impurezas (areia, bagacilhos etc.) e devidamente esterilizado, o caldo está pronto para ser encaminhado para fermentação.

Preparo do mosto: O mosto é uma solução de açúcar cuja concentração foi ajustada de forma a facilitar a sua fermentação. Ele é constituído de uma mistura de méis e caldo, com uma concentração de sólidos de aproximadamente $19-22^{\circ}$ Brix.

Preparo do fermento: $\mathrm{O}$ processo de fermentação mais comumente utilizado nas destilarias do Brasil é o de MelleBoinot, cuja característica principal é a recuperação da levedura através da centrifugação do vinho.

Esta levedura recuperada, antes de 
retornar ao processo fermentativo, recebe um tratamento severo, que consiste em diluição de ácido sulfúrico até alcançar um $\mathrm{pH}=2.5$, ou mais baixo $(\mathrm{pH}=2)$, no caso de haver infecção bacteriana.

Fermentação: Nesta etapa os açúcares são transformados em álcool. As reações ocorrem em tanques denominados dornas de fermentação e os açúcares são transformados em álcool.

Durante a reação, ocorre uma intensa liberação de gás carbônico, a solução é aquecida e ocorre a formação de alguns produtos secundários como: álcoois superiores, glicerol, aldeídos, etc.

Ao terminar a fermentação, o teor médio de álcool nestas dornas é de $7 \%$ a $10 \%$, e a mistura recebe o nome de vinho fermentado.

Centrifugação do vinho: Após a fermentação, o vinho é enviado às centrífugas para a recuperação do fermento. $\mathrm{O}$ concentrado do fermento recuperado, denominado leito de levedura, retorna para tratamento. $\mathrm{O}$ vinho, já com pequenas quantidades de leveduras, é enviado então para a destilação.

Destilação: $O$ etanolpresente neste vinho é recuperado por destilação, processo este que se baseia nos diferentes pontos de ebulição das diversas substâncias voláteis presentes, separandoas.

\section{ESTUDOS DE CASO}

Em qualquer programa de diversificação produtiva na indústria sucroalcooleira a cogeração é um componente importante. Toda a energia utilizada para o processamento da cana, seja térmica ou elétrica, é possível de ser gerada na própria usina, utilizando totalmente ou parcialmente o bagaço resultante da extração do caldo. Portanto, os sistemas analisados utilizam sistemas de cogeração para a satisfação de suas demandas energéticas. Na continuação é realizada uma descrição dos estudos de casos analisados:

\subsection{Sistema (Eletricidade + Etanol)}

ENGEVISTA, V. 16, n. 1, p.58-69, Março 2014
O sistema de produção de eletricidade + etanol (E+E) corresponde a uma destilaria autônoma de produção de etanol com uma capacidade de produção de $41,28 \mathrm{~m}^{3} / \mathrm{h}$.

A demanda de energia elétrica, térmica e mecânica da planta é fornecida por um sistema de cogeração operando com parâmetros de pressão e temperatura de $8,0 \mathrm{MPa}$ e $510{ }^{\circ} \mathrm{C}$, utilizando turbinas de extração/condensação (CEST).

Os principais parâmetros de operação da planta de destilação são apresentados na Tabela 1 .

Tabela 1 - Principais parâmetros de operação da destilaria

\begin{tabular}{|c|c|}
\hline Aquecimento & \\
\hline $\begin{array}{l}\text { Temperatura de entrada do } \\
\text { vapor }\left[{ }^{\circ} \mathrm{C}\right]\end{array}$ & 115 \\
\hline $\begin{array}{l}\text { Temperatura de saída de vapor } \\
{\left[{ }^{\circ} \mathrm{C}\right]}\end{array}$ & 80 \\
\hline Evaporação & \\
\hline $\begin{array}{l}\text { Concentração inicial do caldo } \\
{\left[{ }^{\circ} \text { Brix }\right]}\end{array}$ & 15 \\
\hline $\begin{array}{l}\text { Concentração final do caldo } \\
{\left[{ }^{\circ} \text { Brix }\right]}\end{array}$ & 22 \\
\hline $\begin{array}{l}\text { Temperatura de entrada do } \\
\text { vapor }\left[{ }^{\circ} \mathrm{C}\right]\end{array}$ & 115 \\
\hline $\begin{array}{l}\begin{array}{l}\text { Temperatura } \\
\text { vapor }\left[{ }^{\circ} \mathrm{C}\right]\end{array} \\
\text { ve }\end{array}$ & 69 \\
\hline Resfriamento & \\
\hline $\begin{array}{l}\text { Temperatura de entrada da } \\
\text { água }\left[{ }^{\circ} \mathrm{C}\right]\end{array}$ & 25 \\
\hline $\begin{array}{l}\text { Temperatura de saída da água } \\
{\left[{ }^{\circ} \mathrm{C}\right]}\end{array}$ & 34 \\
\hline $\begin{array}{l}\text { Temperatura de entrada do } \\
\text { caldo }\left[{ }^{\circ} \mathrm{C}\right]\end{array}$ & 68 \\
\hline $\begin{array}{l}\text { Temperatura de saída do caldo } \\
{\left[{ }^{\circ} \mathrm{C}\right]}\end{array}$ & 28 \\
\hline Fermentação & \\
\hline $\begin{array}{l}\text { Concentração de etanol na } \\
\text { saída [\%] }\end{array}$ & 9 \\
\hline Destilação & \\
\hline $\begin{array}{l}\text { Concentração de etanol na } \\
\text { saída [\%] }\end{array}$ & 95 \\
\hline $\begin{array}{l}\text { Temperatura de entrada do } \\
\text { vapor }\left[{ }^{\circ} \mathrm{C}\right]\end{array}$ & 115 \\
\hline $\begin{array}{l}\text { Temperatura de saída do } \\
\text { vapor }\left[{ }^{\circ} \mathrm{C}\right]\end{array}$ & 60 \\
\hline $\begin{array}{l}\text { Consumo específico de vapor } \\
\text { no processo }[\mathrm{kgv} / \mathrm{tc}]\end{array}$ & 368 \\
\hline
\end{tabular}




\subsection{Sistema (Eletricidade + Etanol + Biometanol)}

Para este estudo de caso (E+E+BM), considera-se que a planta de metanol está anexada a uma destilaria de etanol. A capacidade de produção da planta de metanol é estimada em $328 \mathrm{~m}^{3} /$ dia e a planta de etanol em $2594 \mathrm{~m}^{3} /$ dia.

O bagaço, um subproduto do processo de moagem da cana, tem como principais funções, neste sistema:

- Combustível para o sistema de cogeração que visa atender a demanda energética (energia elétrica, térmica e mecânica) das duas plantas;

- Matéria-prima para a produção de metanol pela rota da gaseificação do bagaço.

O sistema de cogeração é baseado em turbinas de extração e condensação, operando com parâmetros de pressão e temperatura de: $10 \mathrm{MPa} / 520^{\circ} \mathrm{C}, 2 \mathrm{MPa} /$ $300^{\circ} \mathrm{C}$.Os principais parâmetros do sistema estão descritos na Tabela 2.

$\mathrm{Na}$ Tabela 3, estão listados os principais dados de consumo e produção da planta de metanol e da destilaria de etanol.
Tabela 2 - Parâmetros do sistema de cogeração

\begin{tabular}{|c|c|}
\hline $\begin{array}{l}\text { Desempenho dos } \\
\text { Equipamentos e Sistema }\end{array}$ & \\
\hline Eficiência da caldeira [\%] & 88 \\
\hline $\begin{array}{l}\text { Eficiência isentrópica da } \\
\text { turbina a vapor [\%] }\end{array}$ & 90 \\
\hline Potência instalada [MW] & 78 \\
\hline Produção de vapor [t/h] & 600 \\
\hline Combustível (Bagaço) & \\
\hline $\begin{array}{l}\text { Bagaço de cana-de-açúcar - } \\
\text { PCI }[\mathrm{kJ} / \mathrm{kg}]\end{array}$ & 7.560 \\
\hline $\begin{array}{l}\text { Teor de umidade do bagaço } \\
{[\%]}\end{array}$ & 50 \\
\hline Sistema de Moagem & \\
\hline $\begin{array}{l}\text { Pressão de entrada do vapor } \\
\text { [Mpa] }\end{array}$ & 2 \\
\hline $\begin{array}{l}\text { Temperatura de entrada do } \\
\text { vapor }\left[{ }^{\circ} \mathrm{C}\right]\end{array}$ & 320 \\
\hline $\begin{array}{l}\text { Pressão do vapor de exaustão } \\
\text { [Mpa] }\end{array}$ & 0,170 \\
\hline $\begin{array}{l}\text { Eficiência isentrópica } \quad \mathrm{da} \\
\text { turbina [\%] }\end{array}$ & 60 \\
\hline $\begin{array}{l}\text { Porcentagem de bagaço } \\
\text { extraído da cana }\end{array}$ & 27 \\
\hline $\begin{array}{l}\text { Capacidade de moagem de } \\
\text { cana-de-açúcar }[\mathrm{t} / \mathrm{h}]\end{array}$ & 1.351 \\
\hline Condições ambientais & \\
\hline Temperatura atmosférica $\left[{ }^{\circ} \mathrm{C}\right]$ & 25 \\
\hline Pressão atmosférica [MPa] & 0,1013 \\
\hline
\end{tabular}

Tabela 3 - Dados de consumo e produção da destilaria de etanol e da planta de biometanol

\begin{tabular}{|l|l|}
\hline $\begin{array}{l}\text { Consumo de vapor total } \\
{[\mathrm{kg} / \mathrm{t} \text { cana }]}\end{array}$ & 369 \\
\hline Produção de metanol $\left[\mathrm{m}^{3} / \mathrm{h}\right]$ & 14 \\
\hline Produção de etanol $\left[\mathrm{m}^{3} / \mathrm{h}\right]$ & 114 \\
\hline $\begin{array}{l}\text { Consumo de energia elétrica da } \\
\text { planta de metanol }[\mathrm{kWh} / \mathrm{kg} \mathrm{de} \\
\text { metanol }]\end{array}$ & 0,25 \\
\hline
\end{tabular}

\section{INDICADORES UTILIZADOS}

Para realizar uma avaliação de desempenho nos dois estudos de caso, foi considerado um sistema de cogeração com bagaço em ambos os estudos, determinando índices de desempenho baseados na Primeira e Segunda Lei da 
Termodinâmica. A seguir são apresentados e definidos alguns deles (Fenget al., 1998):

\subsection{Fator de Utilização de Energia (FUE)}

Conhecido também como eficiência energética ou eficiência da primeira lei, é um indicador baseado no princípio físico da conservação de energia.

$$
F U E=\frac{W+Q_{u}}{m_{\text {comb }} \times P C I}
$$

Onde: PCI do bagaço é de $7.560 \mathrm{~kJ} / \mathrm{kg}$

\subsection{Eficiência elétrica $\left(\eta_{\mathrm{e}}\right)$}

A eficiência de geração de potência não considera a parcela relativa ao calor de processo. A eficiência de geração é definida como:

$$
\eta_{e}=\frac{W}{m_{\text {comb }} \times P C I}
$$

\section{3 Índice de eletricidade excedente ( $\mathrm{I}_{\text {exced}}$ )}

Este índice é a razão do excedente de energia elétrica $\left(W_{\text {exced }}\right)$ pela quantidade de cana moída $(t c)$ em toneladas por hora:

$$
I_{\text {exced }}=\frac{W_{\text {exced }}}{t c}
$$

\subsection{Eficiência exergética ( $\eta_{\text {exerg}}$ )}

Este índice se refere a eficiência calculada de acordo com a segunda lei da termodinâmica. Comparada a equação (4), tanto a energia do calor de processo é substituída pela exergia consumida pelo processo $\left(B_{p}\right)$, quanto a energia do combustível é substituída pela exergia $\left(B_{f}\right)$ :

$$
\eta_{\text {exerg }}=\frac{W+B_{p}}{B_{f}}
$$

\subsection{Eficiência global da $\operatorname{planta}\left(\eta_{\text {global }}\right)$}

A fim de analisar a função produtividade de obtenção de energia e combustíveis a partir da cana-de-açúcar propõe-se dividir o somatório da energia do etanol, da eletricidade excedente e do metanol, pela energia contida na cana-deaçúcar, como mostra a equação a seguir:

$$
\eta_{\text {global }}=\frac{E_{e \tan o l}+W_{\text {exced }}+E_{\text {metanol }}}{E_{c a n a}}
$$

\subsection{Indicadores econômicos}

Tendo em consideração que nos sistemas de poligeração analisados são obtidos múltiplos produtos com valor agregado, é preciso distribuir os custos totais da planta de forma racional e justa entre as diferentes formas de energia produzidas, já que os custos destas vão influenciar diretamente nos preços de cada um dos produtos finais (etanol + eletricidade e etanol + biometanol + eletricidade).

Para se chegar aos custos finais dos biocombustíveis e da eletricidade produzidos é preciso adotar um método justo de partição ou de rateio no sistema para que cada produto tenha um preço que reflita os custos reais envolvidos na sua produção, sem sobrecarregar um ou outro. Porém esta não é uma tarefa fácil, já que o melhor método de alocação de custos em plantas com múltiplos produtos é ainda tema de debates.

Atualmente, diversos autores apontam a exergia como a melhor forma de valorização dos fluxos de energia de uma planta. No caso dos sistemas de cogeração a exergia é a melhor forma de valorização do calor.

Neste trabalho o método de alocação de custos aplicado é baseado na Segunda Lei da Termodinâmica, ou seja, é utilizada uma metodologia de análise termoeconômica que combina o conceito de exergia com aspectos econômicos, na repartição dos custos da planta. Esta metodologia permite alocar os custos dos insumos, assim como os custos de 
investimento, operação e manutenção dos equipamentos para os biocombustíveis (etanol + biometanol) e para a eletricidade em função da exergia efetivamente necessária para produzir cada um.

A alocação de custos nos sistemas de poligeração pode ser direcionada de duas formas diferentes dependendo do objetivo pretendido:

- Alocar todos os custos (cana-deaçúcar, investimento, operação e manutenção, etc.), para se chegar aos custos de produção dos biocombustíveis e da eletricidade durante o período de amortização dos equipamentos.

- Alocar os custos do combustível e da operação e manutenção, para se chegar aos custos de produção dos biocombustíveis e da eletricidade após a amortização dos equipamentos.

No presente estudo foi escolhido o primeiro método de alocação.

\section{Formação de custos}

Um sistema de poligeração pode ser considerado como um sistema ou um conjunto de subsistemas trocando fluxos (massa e energia) entre si e entre eles e o ambiente. Essas informações podem ser traduzidas em fluxos financeiros ou de custos $(C)$.

As equações de custos representam o balanço dos fluxos contábeis tendo no primeiro membro o somatório das entradas e no segundo membro os fluxos de saída.

A composição de custos num sistema de poligeração como um único sistema, pode ser escrita de forma geral pela Equação (10), cujas parcelas estão expressas em unidade financeira pelo tempo $(\$ / h)$.

$$
C_{C}+Z_{P P}=C_{\text {biocombustivel }}+C_{\text {electricidade }}
$$

Como custos de entrada das plantas analisadas, têm-se o custo da cana-deaçúcar $(C c)$, e a parcela $\left(Z_{P P}\right)$ referente aos custos de amortização, operação e manutenção dos equipamentos que compõem as plantas. Como produtos ou saídas da equação têm-se os custos alocados aos biocombustíveis $\left(C_{\text {biocombustivel }}\right)$ e a potência elétrica $\left(C_{\text {eletricidade }}\right)$.

Para calcular os custos de interesse, neste caso aos custos unitários de produção de eletricidade $\left(c_{\text {eletricidade }}\right) \mathrm{e}$ biocombustíveis ( $c_{\text {biocombustiveis }}$ ), expressos em $\$ / \mathrm{kWh}$ e $\$ / 1$, é conveniente que a Equação (10) seja escrita na forma da Equação (11).

$$
\begin{gathered}
C_{C}+Z_{P P}=c_{\text {biocombustivel }} \cdot \dot{V}_{\text {biocombustivel }} \\
+c_{\text {eletricidade }} \cdot P_{\text {eletrica }}
\end{gathered}
$$

Onde $\mathrm{V}_{\text {biocombustível é a produção }}$ horária de biocombustível e $\mathrm{P}_{\text {eletrica }}$ é a potência elétrica líquida expressa em $\mathrm{kW}$ e $\mathrm{m}^{3} / \mathrm{h}$, respectivamente.

A equação (11) não é ainda suficiente para resolver o problema de alocação de custos em sistemas de poligeração, já que são duas incógnitas ( $c_{\text {biocombustiveis }} \mathrm{e}$ $c_{\text {eletricidade }}$ ) que não têm relação direta entre si. $O$ primeiro é um fluxo puramente volumétrico (ou mássico) enquanto o segundo é um fluxo energético.

Para obter o conjunto de equações auxiliares que permitam determinar os custos unitários dos principais produtos foi utilizado o equacionamento matemático proposto por Santos et al. (2006) e Frangopoulos (1994).

Os custos dos equipamentos que compõem as plantas dos dois estudos de caso analisados foram obtidos através de empresas fabricantes de equipamentos nacionais. Outros foram estimados através da Equação (12), que permite determinar os custos dos equipamentos com capacidade diferente a aqueles dos quais se possui informação. Neste caso, através do conhecimento das características técnicas e do custo destes equipamentos e de posse das características dos atuais da instalação, foi possível determinar o custo dos equipamentos existentes nas plantas consideradas. 


$$
\frac{\text { Custo }_{a}}{\text { Custo }_{b}}=\left(\frac{\text { Capacidade }_{a}}{\text { Capacidade }_{b}}\right)^{R}
$$

Onde R é o Fator de escala (permite estimar o custo de um equipamento em caso que este possua dados de custo relativos à outra capacidade ou tamanho).

Durante o levantamento dos preços dos equipamentos, não estavam disponíveis todos os itens que podem ser incluídos na análise monetária, sendo necessário o estabelecimento de percentuais em relação ao preço de aquisição do equipamento. As porcentagens estão listadas na Tabela 4.

Tabela 4 - Valores percentuais empregados na estimativa dos custos dos equipamentos

\begin{tabular}{|l|c|}
\hline Custos & $\begin{array}{c}\text { Porcentuais } \\
\text { [\%] }\end{array}$ \\
\hline Custo de Instalação & 20 \\
\hline $\begin{array}{l}\text { Custo das } \\
\text { Tubulações }\end{array}$ & 10 \\
\hline $\begin{array}{l}\text { Custo da } \\
\text { Instrumentação e } \\
\text { Controle }\end{array}$ & 6 \\
\hline $\begin{array}{l}\text { Custo de } \\
\text { Equipamentos } \\
\text { Elétricos e Materiais }\end{array}$ & 10 \\
\hline $\begin{array}{l}\text { Custo das } \\
\text { Construções Civis } \\
\text { (Edifícios) }\end{array}$ & 5 \\
\hline $\begin{array}{l}\text { Custo de Operação } \\
\& \text { Manutenção }\end{array}$ & 15 \\
\hline
\end{tabular}

$\mathrm{O}$ investimento econômico requerido pelos sistemas de poligeração $(E+E)$ e $(\mathrm{E}+\mathrm{E}+\mathrm{BM})$ é apresentado na Tabela 5.

Tabela 5 - Investimento econômico requerido pelos sistemas de poligeração

\begin{tabular}{|l|c|}
\hline Sistema & $\begin{array}{c}\text { Investimento } \\
\text { [Milhões US\$] }\end{array}$ \\
\hline $\mathrm{E}+\mathrm{E}$ & $55.022 .045,95$ \\
\hline $\mathrm{E}+\mathrm{E}+\mathrm{BM}$ & $163.545 .468,92$ \\
\hline
\end{tabular}

O custo da planta de produção de metanol a partir de bagaço foi determinado com base no trabalho realizado porHamelinck e Faaij (2002). A taxa de juros considerada foi de $8 \%$

ENGEVISTA, V. 16, n. 1, p.58-69, Março 2014 enquanto o período de amortização dos equipamentos foi de 10 anos.

\section{RESULTADOS}

\subsection{Avaliação energética}

Com base nas equações e definições apresentadas no item anterior, os principais resultados dos estudos de caso considerados (etanol + eletricidade; etanol + metanol + eletricidade) estão apresentados na Tabela 6.

Tabela 6 - Índices de desempenho baseados na primeira e segunda lei da termodinâmica

\begin{tabular}{|l|c|c|}
\hline & \multicolumn{2}{|c|}{ Estudo de caso } \\
\hline Índice & $\begin{array}{c}\text { Sistema } \\
\text { E + E }\end{array}$ & $\begin{array}{c}\text { Sistema } \\
\mathbf{E}+\mathbf{E}+\mathbf{B M}\end{array}$ \\
\hline FUE $(\%)$ & 67 & 79 \\
\hline$\eta_{\mathrm{e}}(\%)$ & 17 & 14 \\
\hline $\begin{array}{l}\text { Iexced } \\
(\mathrm{kWh} / \mathrm{t} \text { cana })\end{array}$ & 89,2 & 54,9 \\
\hline$\eta_{\text {exerg }}(\%)$ & 24 & 22 \\
\hline$\eta_{\text {global }}(\%)$ & 42 & 47 \\
\hline
\end{tabular}

$\mathrm{Na}$ Tabela 6 pode ser observado que o fator de utilização de energia (FUE) é $15 \%$ maior para o sistema $\mathrm{E}+\mathrm{E}+\mathrm{BM}$ em relação ao sistema $\mathrm{E}+\mathrm{E}$, no entanto este indicador não permite a obtenção de conclusões adequadas sobre $\mathrm{o}$ desempenho dos sistemas por ser a eletricidade e o vapor energia com qualidades diferentes.

Já a eficiência elétrica $\left(\eta_{\mathrm{e}}\right)$, a eficiência exergética $\left(\eta_{\text {exerg }}\right)$ e o índice de eletricidade excedente $\left(\mathrm{I}_{\text {exced }}\right)$ foram $3 \%$, $2 \%$ e $34.3 \%$ superiores no sistema $\mathrm{E}+\mathrm{E}$ em relação ao sistema $\mathrm{E}+\mathrm{E}+\mathrm{BM}$. Isto se justifica no fato que o processo de produção de biometanol demanda um alto consumo de energia elétrica, no processo de síntese que exige a pressurização do gás de síntese para favorecer as reações químicas de produção de biometanol. Por outro lado é necessária a realização de extrações de vapor em alta pressão e temperatura no sistema de cogeração do sistema $\mathrm{E}+\mathrm{E}+\mathrm{BM}$ para atender a demanda térmica dos processos de gaseificação de bagaço e síntese de 
metanol da planta de produção de metanol.

Porém com relação á eficiência global $\left(\eta_{\text {global }}\right)$, o sistema $\mathrm{E}+\mathrm{E}+\mathrm{BM}$ apresenta uma porcentagem $5 \%$ maior que o $\mathrm{E}+\mathrm{E}$. Isto devido ao maior aproveitamento energético da cana-de-açúcar, na obtenção de 3 produtos (eletricidade, etanol e biometanol), ao contrário do outro sistema, onde produz somente 2 (eletricidade e etanol).

Portanto, em termos de aproveitamento energético global, o sistema $\mathrm{E}+\mathrm{E}+\mathrm{BM}$ apresenta-se como a melhor alternativa.

As vantagens energéticas oferecidas nos sistemas de poligeração analisados serão complementadas com a realização de uma análise econômica dos sistemas considerados

\subsection{Avaliação econômica}

$\mathrm{Na}$ Figural é apresentada uma representação esquemática do processo de distribuição e formação de custos no sistema $\mathrm{E}+\mathrm{E}$ de acordo como o propósito produtivo de cada subsistema que compõe a planta e a exergia consumida em cada etapa.

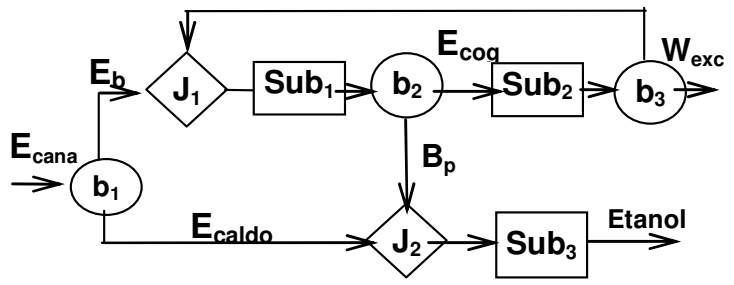

Figura 1. Alocação de custos no sistema $\mathrm{E}+\mathrm{E}$ em função dos fluxos exergéticos da planta.

O subsistema $1 \quad\left(\mathrm{Sub}_{1}\right)$ da Fig. 1 representa todos os componentes da planta que injetam exergia no ciclo (bombas, caldeiras, desaereador,etc.), enquanto $\mathrm{o}$ subsistema $2 \quad\left(\mathrm{Sub}_{2}\right)$ representa alguns componentes que consomem exergia no ciclo (turbogeradores, condensadores, acionadores mecânicos), finalmente $\mathrm{o}$ subsistema $3\left(\mathrm{Sub}_{3}\right)$ representa as etapas do processo de produção de etanol (aquecimento, evaporação, resfriamento, fermentação e destilação). Desta forma é possível conhecer o processo de formação de custos dos principais produtos da planta.

A equação (11) mostra que numa planta de poligeração os custos dos principais produtos estão um em função do outro, portanto, é necessário alocar o custo da cana de maneira proporcional no sistema global para evitar sobrecarregar o custo de um dos produtos obtidos. Isto implica que no caso de considerar que o bagaço que entra no sistema de cogeração tem um custo zero por ser um subproduto do processo, o custo da cana será carregado ao caldo obtido na moenda, tendo como conseqüência que o etanol produzido terá um custo maior ao custo real de produção enquanto a eletricidade terá um custo menor.

A Figura2 mostra o resultado dos custos monetários unitários para os principais produtos do sistema $\mathrm{E}+\mathrm{E}$.

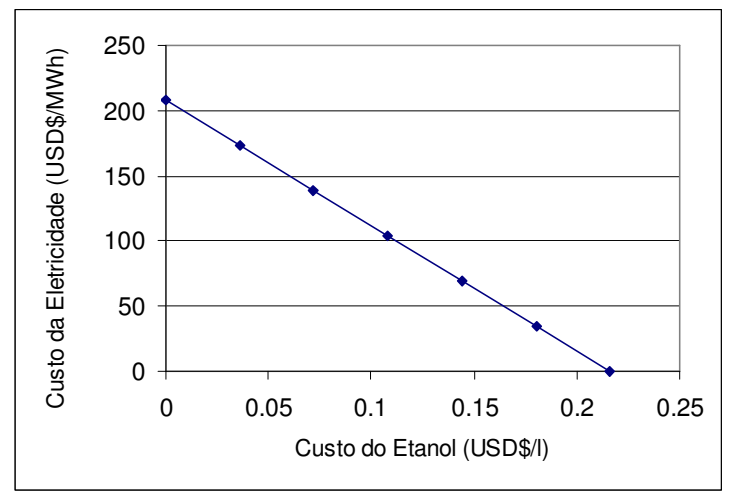

Figura 2. Custo monetário unitário dos principais produtos do sistema $\mathrm{E}+\mathrm{E}$.

Observa-se na Figura2 que os custos da eletricidade estão em função dos custos do etanol e vice-versa. O que significa que na medida em que o custo de mercado do etanol aumenta, a produção do mesmo compensa economicamente a diminuição eventual no custo de compra da eletricidade excedente comercializada pelo sistema. De igual forma preços altos de compra de eletricidade excedente do sistema compensam economicamente diminuições no preço do mercado e do etanol.

Semelhante ao apresentado na 
Figura1, na Figura3 é apresentada uma representação esquemática do processo de distribuição e formação de custos no sistema $\mathrm{E}+\mathrm{E}+\mathrm{BM}$.

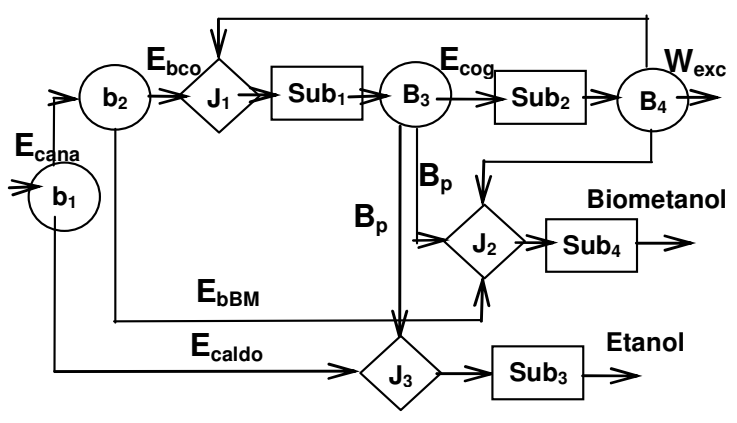

Figura3. Alocação de custos no sistema

$\mathrm{E}+\mathrm{E}+\mathrm{BM}$ em função dos fluxos exergéticos da planta.

$\mathrm{Na}$ Figura3, o subsistema $1 \quad\left(\mathrm{Sub}_{1}\right)$ representa todos os componentes da planta que injetam exergia no ciclo (bombas, caldeiras, desaereador,etc.), enquanto $\mathrm{o}$ subsistema $2 \quad\left(\mathrm{Sub}_{2}\right)$ representa alguns componentes que consomem exergia no ciclo (turbogeradores, condensadores, acionadores mecânicos), o subsistema 3 $\left(\mathrm{Sub}_{3}\right)$ representa as etapas do processo de produção de etanol (aquecimento, evaporação, resfriamento, fermentação e destilação) e finalmente o subsistema 4 $\left(\mathrm{Sub}_{4}\right)$ representa as etapas do processo de produção de biometanol (prétratamento, gaseificação e síntese do metanol). Desta forma é possível descrever o processo de formação de custos dos principais produtos da planta que a diferença do sistema $\mathrm{E}+\mathrm{E}$ apresenta três produtos principais, neste caso os resultados da avaliação econômica são apresentados na Figura4.

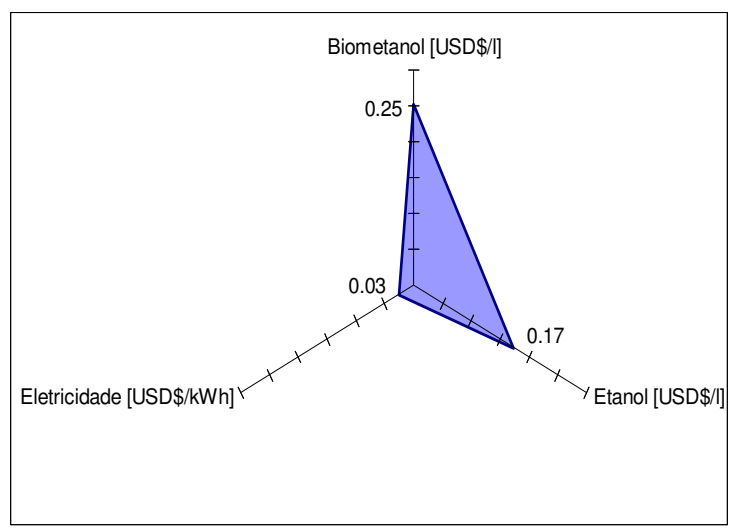

Figura4. Custo monetário unitário dos principais produtos do sistema $\mathrm{E}+\mathrm{E}+\mathrm{BM}$

$\mathrm{Na}$ Figura4 observa-se que para um custo de produção de eletricidade de 0,03 USD $\$ / \mathrm{kWh}$ e um custo de produção do etanol de 0,17 USD $\$ / 1$ o custo de produção do biometanol é de 0,25 USD\$/1, o qual é $24 \%$ superior ao preço do metanol fóssil obtido do gás natural que apresenta um preço de 0,19 USD \$/1 Methanex (2009).

No entanto, tendo em conta que numa planta de poligeração o custo de mercado de um produto compensa economicamente a produção dos outros, na medida em que aumentem os preços do mercado do etanol o da eletricidade comercializada compensaram economicamente os custos de produção do biometanol, permitindo assim a viabilidade econômica da planta.

De igual forma esperam-se nos próximos anos, a introdução de melhoras nas etapas produtivas do processo de produção de biometanol que diminuíram ainda mais os custos de produção através da rota tecnológica da gaseificação.

Tendo em consideração que a determinação dos investimentos de alguns equipamentos foi estimada de acordo com a Equação (12) e de algumas referências bibliográficas os resultados podem apresentar uma margem de erro aproximadamente $30 \%$.

\section{CONCLUSÕES}

Neste trabalho foi realizada uma análise econômica e energética de dois sistemas de poligeração (um com produção de eletricidade e etanol e outro produzindo biometanol, etanol e eletricidade). Para a análise energética, baseou-se em indicadores relacionados com a Primeira e Segunda Lei da Termodinâmica, quanto para a análise econômica, foi selecionado o método de alocação de custos baseado na Segunda Lei da Termodinâmica (Análise Termoeconômica).

Os principais resultados obtidos neste estudo mostram: 
A implementação de sistemas de poligeração nas cadeias produtivas de biocombustíveis permitiram o aumento na sustentabilidade dos mesmos ao incrementar a eficiência na utilização dos recursos demandados pelo processo dado o melhor aproveitamento energético global da planta.

Economicamente foi demonstrado que nas plantas de poligeração, o custo econômico de um produto no mercado pode compensar a diminuição do custo de outro produto, o que permite uma maior segurança na rentabilidade econômica da planta.

A integração de uma planta de produção de metanol a partir de bagaço a uma destilaria convencional mostra-se como uma alternativa de diversificação da produção de biocombustíveis no Brasil.

Espera-se que nos próximos anos, o desenvolvimento tecnológico das tecnologias de produção de biometanol pela rota da gaseificação diminua os custos de produção deste biocombustível, o que aumentaria a viabilidade econômica de plantas de biometanol anexas, nas condições do setor sucroalcooleiro brasileiro.

\section{REFERÊNCIAS}

CHEN, B., JIN, H.G.,GAO, L.2006. Study of DME/power individual generation and polygeneration.Journal of Engineering Thermophysics, 27, 721724.

CIFRE, P. G., BRAD, O. 2007. Renewable hydrogen utilization for the production of methanol.Energy Conversion and Management, 48, 519527.

FENG, X.,CAI, Y., QIAN, L. 1998. A new performance criterion for cogeneration system.Energy Conversion and Management, 39, 1607-1609.

FRANGOPOULOS, C.A. 1994. Application of the thermoeconomic functional approach to the CGAM problem. Energy, 19, n 3, 323-342.

HAMELINCK, C. N., FAAIJ, A. C. 2002. Future prospect for production of methanol and hydrogen from biomass.Journal of Power Sources, 111, 1-22.

MA, L. W., NI, W. D., LI, Z., REN, T. J. 2004. Analysis of the polygeneration system of methanol and electricity based on coal gasification.Power Engineering, 24, 451-456.

MAIYA, P. S., ANDERSON, T. J., MIEVILLE, R. L., DUSEK, J. T., PICCIOLO, J. J., BALACHANDRAN, U. 2000. Maximizing $\mathrm{H}_{2}$ production by combined partial oxidation of $\mathrm{CH}_{4}$ and water gas shift reaction.Applied Catalysis A:General, 196, 65-72.

METHANEX, Methanol Price Sheet 2009 [Online]. Available: www.methanex.com/products/methanolpr ice.html [Accessed 10 June 2009].

OULLETTE, N.,ROGNER, H. H., SCOTT, D. S. 1995. Hydrogen from remote excess hydroelectricity. Part II: Hydrogen peroxide of biomethanol. Int J. Hydrogen Energy, 20, 873-880.

SANTOS, J.J., NASCIMENTO, M.A., LORA, E.E. 2006.On the thermoeconomic modeling for cost allocation in a dual-purpose power and desalination plant. In Proceedingof ECOS 2006, Crete, Greece.

SANTOS, M. M. 2004. Avaliação da expansão da produção de etanol no Brasil, Centro de Estudos Estratégicos, Brasília.

YAMASHITA, K., BARRETO, L. 2005. Energyplexes for the $21^{\text {st }}$ century: coal gasification for co-producing hydrogen, electricity and liquid fuels. Energy, 30, pp. 2453-2473. 